Overall, it affects the patients' self-image and the patients' independence may be lost.

Conclusions: $\mathrm{HAQ}$ questionnaire is relevant in the term of defining the areas in everyday life, where the patients have problems. But the questionnaire does not contribute to elucidate the implications for the patient, which probably is the reason why many patients do not find the meaning with the questions. To evaluate the everyday living with and treatment of arthritis embedding the patient values, patient involvement in designing questionnaires is vital

References:

[1] Kvien TK. Epidemiology of disability in Rheumatoid arthritis. Rheumatology 2002;41:121-123.

[2] Seror R et al. Measure of function in rheumatoid arthritis: individualised or classical scales? Ann Rheum Dis. 2010;69: 97-101.

[3] Lütze $U$, Archenholtz B. The impact of arthritis on daily life with the patient perspective in focus. Scand J Caring Sci 2007;21(1):64.

[4] Hughes JG. Exploring the impact of rheumatoid arthritis on patients' lives. International journal of therapy and rehabilitation $2009-11-01 ; 16(11): 594$; 594-601; 601.

Disclosure of Interest: None declared

DOI: 10.1136/annrheumdis-2017-eular.2153

\section{SAT0745-HPR IMPACT OF AN INTERDISCIPLINARY INTERVENTION ON RA PATIENTS}

I. Fortin ${ }^{1}$, H. Sylvain ${ }^{2}$, F. Banville ${ }^{3}$, B. Lovisi ${ }^{4}$, S.V. Rochefort ${ }^{1}$. ${ }^{1}$ Research, CISSS du Bas-Saint-Laurent/Hôpital Régional de Rimouski; ${ }^{2}$ Sciences infirmières; ${ }^{3}$ Research, Université du Québec à Rimouski, Rimouski; ${ }^{4}$ Rheumatology, CISSS du Saguenay-Lac-St-Jean/Hôpital de Chicoutimi, Chicoutimi, Canada

Background: Rheumatoid arthritis (RA) impairs patient function and quality of life (QoL) which, in turn, may lead to invalidity and chronicity of pain1. Even though a variety of effective treatments are available, there is still a significant portion of patients not attaining remission, of whom many continue to report moderate to high pain and fatigue despite low levels of inflammation1. Interdisciplinary interventions are promising approaches that may complement the medication effect on patient well-being and disease control, however previous studies on the effectiveness of interdisciplinary interventions seem conflicting.

Objectives: The primary objective is to compare the DAS28 response between patients receiving an interdisciplinary intervention (Intervention group) and patients followed under standard rheumatologic practice (Control group). Secondary objectives are to compare patient-reported outcomes such as pain, fatigue, general health, and patient empowerment between the two groups.

Methods: Prospective quasi-experimental, matched cohort (age, gender) study. Adult patients with a diagnosis of RA and DAS28 (CRP) $>2.6$ are eligible for the study. The Intervention group $(n=28)$ benefits from interdisciplinary team intervention with the following professionals: rheumatologist, nurse, physiotherapist, social worker, kinesiologist, occupational therapist, and nutritionist. The Control group $(n=32)$ receives a conventional rheumatologist-nurse intervention. Both groups see the rheumatologist approximately three times over 12 months. Interdisciplinary meetings take place in the hospital setting with the patients at Month 0 and Month 12 and without them at Month 6. The following outcome measures are used at each visit to the rheumatologist: patient pain, fatigue (Multidimensional Assessment of Fatigue; MAF), disability (HAQ), quality of life (SF-36), patient empowerment (Patient Activation Measure; PAM13), and patient satisfaction (QSC-F; only in Intervention group at Month 12).

Results: A total of 28 patients were enrolled in the Intervention group and 32 in the Control group without any significant differences in demographics or disease parameters with the exception of disease duration which was significantly higher in the Intervention group (10.9 vs. 5.8 years; $p=0.021)$. Within 6 months of treatment, clinically important and statistically significant $(p<0.01)$ improvements in DAS28 were observed in both groups which were maintained until 12 months. Overall, at 12 months, DAS28 response was comparable between groups $(68 \%$ vs. $63 \% ; \mathrm{p}=0.140)$. However, when looking at patients with established RA ( $75 \%$ vs. $66.7 \% ; p=0.039$ ) and patients with low to moderate disease activity at baseline $(73.9 \%$ vs. $56.5 \%$; $p=0.035)$, a higher response rate was observed in the Intervention group. No statistical differences were observed in the remaining outcomes between groups.

Conclusions: The results of the current study suggest that interdisciplinary interventions may be useful in conferring benefits on patient well-being and disease control that are above and beyond those resulting from medications. This seems to be particularly true among patients with established disease. Additional analyses looking into the exact mechanism of action of such interventions are required.

Disclosure of Interest: I. Fortin Grant/research support from: grant UCB, H. Sylvain Grant/research support from: Grant UCB, F. Banville Grant/research support from: UCB, B. Lovisi: None declared, S. V. Rochefort Grant/research support from: UCB

DOI: 10.1136/annrheumdis-2017-eular.6332

\section{SAT0746-HPR TOWARDS MEASUREMENT OF PERSON-CENTERED CARE OUTCOMES IN OUTPATIENT NURSE-LED CLINICS}

V. Bala $^{1,2}$, B. Fridlund ${ }^{3}$, K. Forslind ${ }^{4,5}$, B. Svensson ${ }^{6}$, P. Hagell ${ }^{7} .{ }^{1}$ Department of Medicine, Section of Rheumatology, Helsingborg; ${ }^{2}$ Department of Health Science, Lund University, Lund; ${ }^{3}$ School of Health and Welfare, Jönköping University, Jönköping; ${ }^{4}$ Department of Clinical Science, Section of Rheumatology, Lund University; ${ }^{5}$ Department of Research and Education, Helsingborg's Hospital, Helsingborg; ${ }^{6}$ Section of Rheumatology at the Institution of Clinical Science, Lund University, Lund; ${ }^{7}$ The PRO-CARE Group, School of Health and Society, Kristianstad University, Kristianstad, Sweden

Background: Person-centered care (PCC) is increasingly emphasized as a key component of effective illness management and of developing high quality of care. Despite considerable progress of PCC in many areas of care there is currently a gap and a need for means to assess PCC practice in outpatient care. In rheumatology, PCC is considered an unmet need and further development and evaluation of this approach to care is thus of high priority.

Objectives: To develop an instrument for measuring person-centered care from the perspective of the person with rheumatoid arthritis (RA) in nurse-led outpatient clinics.

Methods: A conceptual framework of PCC in the outpatient context and focusing on the meeting between the person with RA and the nurse and on the patient as an active care partner was undertaken. Based on this framework, qualitative interviews $(1,2)$ and a literature review, a 35 -item questionnaire was proposed and qualitatively tested regarding acceptability and content validity among 50 persons with RA attending a nurse-led outpatient clinic. Two versions of the questionnaire were tested: one using four response categories $(0=$ Totally disagree; $3=$ Completely agree), and one using two response categories ( $0=$ Disagree; $1=$ Agree). Content validity was estimated by calculating Content Validity Index of the individual items (I-CVI) and of the overall instrument (S-CVI).

Results: Respondents found the items easy to understand $(77 \%)$ and relevant $(93 \%)$. Seventy-three percent of the respondents preferred the questionnaire version with four response categories. This version took a mean (SD) of 5.3 (2.5) minutes to complete. I-CVI values ranged from 0.87 to 1.00 and S-CVI was 0.94 . About $80 \%$ of the respondents considered some items to be redundant. This resulted in a reduced 24 -item draft questionnaire that yield a total score between $0-72$.

Conclusions: A preliminary 24-item patient-reported PCC questionnaire was developed. Psychometric testing is needed for validation of this tool before implementation.

\section{References:}

[1] Bala SV, Samuelson K, Hagell P, Svensson B, Fridlund B, Hesselgard K (2012). The experience of care at nurse-led rheumatology clinics. Musculoskeletal Care 10: 202-211.

[2] Bala SV, Samuelson K, Hagell P, Fridlund B, Forslind K, Svensson B, Thomé B (2016). Journal of Clinical Nursing; doi: 10.1111/ jocn.13691.

Disclosure of Interest: None declared

DOI: 10.1136/annrheumdis-2017-eular.6530

\section{SAT0747-HPR CAN ACHIEVING SUSTAINED DAS REMISSION PREVENT PROGRESSION OF SUB- CLINICAL ATHEROSCLEROSIS? A PROSPECTIVE COHORT STUDY IN EARLY RHEUMATOID ARTHRITIS (ERA)}

W.Y. Mak ${ }^{1}$, Q. Shang ${ }^{1}$, X.S.L. Lau ${ }^{1}$, A.P.-W. Lee ${ }^{1}$, C.M. Yu ${ }^{1}$, E.K.-M. Li ${ }^{1}$, P.C.H. Wong ${ }^{2}$, L.H.P. Tam ${ }^{2}$, K.Y. Kwok ${ }^{3}$, M.L. Yip ${ }^{4}$, H.T. Pang ${ }^{5}$, V.W.-N. Lao ${ }^{5}$, I.C.-W. Yim ${ }^{6}$, L.S. Tam ${ }^{1} .{ }^{1}$ Dept of Medicine and Therapeutics, The Chinese University of Hong Kong; ${ }^{2}$ Dept of Medicine and Therapeutics, Prince of Wales Hospital; ${ }^{3}$ Dept of Medicine, Queen Elizabeth Hospital; ${ }^{4}$ Kwong Wah Hospital, Hong Kong, Hong Kong; ${ }^{5}$ Dept of Medicine and Geriatrics, Kwong Wah Hospital; ${ }^{6}$ Dept of Medicine, Tseung Kwan O Hospital, Hong Kong, Hong Kong

Background: Patients with rheumatoid arthritis (RA) have higher incidence of cardiovascular disease (CVD) and prevalence of arterial stiffness (AS) due to underlying inflammation. Effective immunosuppression using anti-TNF was shown to improve AS in early RA (ERA) patients. Whether it is a specific effect by blocking the TNF $\alpha$ pathway or suppression of inflammation remains uncertain. While achieving Disease Activity Score in 28joints (DAS) remission was associated with significant benefits in articular disease, its effect on co-morbidities such as CVD risk is uncertain.

Objectives: To investigate the effect of achieving sustained DAS remission on AS.

Methods: This randomized control trial investigates the effect of 2 tight-control treatment strategies aiming 1.Simplified disease activity score [SDAl $\leq 3.3$ ] or 2.minimal disease activity [DAS <2.6] on AS in ERA patients.120 patients with active disease (DAS $\geq 3.2$ ), symptoms onset $<2$ years and bDMARDs naive were recruited and received 1-year treatment. Treatment are adjusted based on the standardized protocol every 3month aiming at either 1 of the 2 targets. AS is measured by branchial-ankle pulse wave velocity (baPWV) using a dedicated tonometry system (Omron VP-2000).

Results: In the interim analysis, results of 100 patients [male (23.0\%); $52.8 \pm 13$ years] completed 1year follow-up were analyzed. No significant differences between groups in clinical features, DMARD use and baPWV at month12 